\title{
(6) OPEN ACCESS \\ Optical coherence tomography angiography in exudative age-related macular degeneration: a predictive model for treatment decisions
}

\author{
Florence Coscas, ${ }^{1,2}$ Marco Lupidi, ${ }^{1,3}$ Jean François Boulet, ${ }^{4}$ Alexandre Sellam, ${ }^{4}$ \\ Diogo Cabral, ${ }^{1,5,6}$ Rita Serra, ${ }^{7}$ Catherine Français, ${ }^{1}$ Eric H Souied, ${ }^{2}$ Gabriel Coscas ${ }^{1,2}$
}

\begin{abstract}
${ }^{1}$ Centre Ophtalmologique de
I'Odeon, Paris, France

2Department of Ophthalmology,

Centre Hospitalier

Intercommunal de Creteil

University Paris Est Creteil

Creteil, France

${ }^{3}$ Section of Ophthalmology,

Department of Biomedical and

Surgical Sciences, University

of Perugia, S. Maria della

Misericordia Hospital, Perugia,

Italy

${ }^{4}$ Paris VI University, UPMC, Paris, France

${ }^{5}$ Retina Department, Instituto de Oftalmologia Dr Gama Pinto, Lisboa, Portugal

${ }^{6}$ NOVA Medical School,

Universidade Nova de Lisboa,

Lisboa, Portugal

Department of Surgical

Sciences, Eye Clinic, University

of Cagliari, Cagliari, Italy
\end{abstract}

Correspondence to

Dr Florence Coscas, Centre

Hospitalier Intercommunal de

Créteil, Université Paris Est,

Créteil 94010, France; coscas.

f@gmail.com

FC and ML contributed equally.

$\mathrm{FC}$ and $\mathrm{ML}$ are joint first authors.

Received 12 August 2018

Revised 3 October 2018

Accepted 5 November 2018

Published Online First

22 November 2018

\section{Check for updates}

(c) Author(s) (or their employer(s)) 2019. Re-use permitted under CC BY-NC. No commercial re-use. See rights and permissions. Published by BMJ.

To cite: Coscas F, Lupidi M,

Boulet JF, et al.

Br J Ophthalmol

2019:103:1342-1346.

\section{ABSTRACT}

Aims To evaluate on optical coherence tomography angiography (OCT-A), the predictive role of different qualitative findings of choroidal neovascularisations (CNV) in assessing the status of exudative age-related macular degeneration (eAMD) and to develop a potential model to predict the CNV activity.

Methods Retrospective review of the multimodal imaging records of patients with eAMD obtained during treatment for type 1 or type 2 CNV. The qualitative analysis of CNVs on OCT angiograms assessed the presence or absence of tiny branching vessels, loops, peripheral anastomotic arcades and choriocapillaris hypointense halo. These findings were then correlated with those of structural OCT scans. A score forecast was built and validated.

Results One hundred and twenty-six eAMD eyes were enrolled in the study. Exudation was observed in 90 eyes $(71 \%)$ on structural OCT. The qualitative OCT-A analysis revealed: tiny branching vessels in $82.5 \%$ of the cases, vascular loops in $81.7 \%$, peripheral anastomotic arcades in $66.7 \%$ and choriocapillaris hypointense halo in $54.8 \%$. In the univariate analysis, each OCT-A parameter showed a statistically significant correlation with exudation on structural OCT $(p<0.001)$. The overall analysis demonstrated a sensitivity of $96.7 \%$ and a positive predictive value of $87.9 \%$. In the multivariate analysis, a model with four criteria predicted an exudative lesion in $97.6 \%$ of cases and one with two criteria (tiny branching vessels and peripheral anastomotic arcades) in $71.2 \%$.

Conclusions The presence of tiny branching vessels and a peripheral anastomotic arcade appears to predict the lesion activity with a good accuracy and the model based on four criteria enables optimal decisions regarding retreatment in $\mathrm{AMD}$.

\section{INTRODUCTION}

Age-related macular degeneration (AMD) is one of the leading causes of visual impairment in older adults in industrialised countries. ${ }^{1}$ Choroidal neovascularisation $(\mathrm{CNV})$ is a characteristic feature of exudative AMD (eAMD) and is characterised by the growth of abnormal blood vessels from the choroid through the Bruch's membrane. ${ }^{2}$

Fluorescein angiography (FA) is currently the gold standard imaging modality for diagnosing and classifying CNVs whereas indocyanine green angiography (ICGA) is used to identify the entire extent of the lesion. ${ }^{3}$ However, small retinal vessels and prominent vessels are often concealed by late-phase hyperfluorescence and this may compromise the precise assessment of vascular features. In addition, these techniques use intravenous dyes that can result in systemic side effects including anaphylaxis. ${ }^{4}$

Despite these limitations, FA is routinely used in conjunction with structural optical coherence tomography (OCT) for the diagnosis and follow-up of patients suffering from AMD. OCT findings are surrogate markers of leakage and are commonly used as treatment or retreatment parameters when evaluating the efficacy of anti-vascular endothelial growth factor (VEGF) agents in clinical settings as well as in current clinical trials. ${ }^{5}$

OCT angiography (OCT-A) provides a clear, depth-resolved visualisation of the retinal and choroidal microvasculature. ${ }^{67}$ Swept-source OCT-A allows an improved visualisation of the choroid, resulting in detailed visualisation of the CNV especially the sub-retinal pigmented epithelium (RPE) component of the neovascularisation. ${ }^{89}$

Previous reports have suggested that OCT-A detects CNVs with the same sensitivity as FA. ${ }^{10} 11$ Moreover, the correspondences between different $\mathrm{CNV}$ patterns and the status of neovascular lesions have also been evaluated. However, to the best of our knowledge, no previous studies have addressed the decision efficacy of treating eAMD based solely on OCT-A findings.

The aim of this study was to evaluate the predictive role of different OCT-A qualitative findings in assessing the status of CNVs and to develop a potential predictive scheme based on these morphological features.

\section{MATERIALS AND METHODS}

Retrospective cross-sectional review of the medical records of consecutive patients observed at the Centre Ophtalmologique de l'Odeon between January 2017 and April 2017. This study was conducted in accordance with the tenets of the Declaration of Helsinki and French legislation.

\section{Study population}

Eligible patients had to meet the following parameters: (1) diagnosis of eAMD through multimodal imaging (FA, ICGA and structural OCT); (2) type 1 or type $2 \mathrm{CNV}^{12}$; and (3) at least one intravitreal anti-VEGF injection (IVI) within the last 3 months and a minimum interval of 1 month between the last IVI and the OCT-A examination. Patients with 
CNV fibrotic scarring, type $3 \mathrm{CNV}$, polypoidal choroidal vasculopathy, retinal vascular or inflammatory diseases complicated with CNV or poor-quality images on OCT-A (signal strength index lower than 80) were excluded.

The retreatment protocol was 'pro-re-nata' and it was based on structural OCT findings. All patients have received a loading dose of three monthly injections of anti-VEGF and then followed up monthly; an IVI was given only in case of evidence of exudation on structural OCT scans. ${ }^{13}$

\section{Image analysis acquisition}

A macular volume scan (49 B-scans within a $30^{\circ} \times 20^{\circ}$ area) centred on the fovea was acquired with a spectral domain OCT (Spectralis OCT2, Heidelberg Engineering, Heidelberg, Germany). ${ }^{14}$ OCT-A was obtained with a swept-source OCT device (Triton SS-OCT, Topcon, Tokyo, Japan) on a scanning area of $4.5 \times 4.5 \mathrm{~mm}$ and an automatically segmented slab of $30 \mu \mathrm{m}$ (shaped on RPE profile) was set immediately above the RPE and then moved towards the Bruch's membrane in order to detect flow signals due to $\mathrm{CNVs} .{ }^{11} \mathrm{~A}$ manual correction of the segmented boundaries was performed in a limited number of acquired OCT angiograms (less than 5\%), especially in case of severe outer retinal/RPE disruption. This process was done by a senior retina specialist (FC), before the OCT-A qualitative grading, in case of incorrect RPE automatic delineation and was performed on structural, cross-sectional OCT scans.

\section{Data sources}

All the patients underwent a comprehensive eye examination including best corrected visual acuity (BCVA) using the ETDRS visual chart, retinal photography of the fundus, structural OCT and OCT-A. The patients' baseline characteristics included age, gender, follow-up duration and number of previous IVI of antiVEGF agents.

\section{Variables}

Structural OCT findings were used to guide the decision to retreat. Two different parameters were assessed: presence of subretinal, intraretinal or sub-RPE fluid (vs absence) and central macular thickness (CMT, expressed in microns). An exudative lesion was defined by the presence of subretinal and/or intraretinal (or sub-RPE) fluid and macular thickening on structural OCT. $^{14} 15$

The evaluation of OCT angiograms was performed in order to delineate the morphological qualitative features of a CNV. We considered four OCT-A criteria ${ }^{11}$ to qualitatively describe a neovascular lesion: (1) presence of tiny branching vessels (thin, tangled capillaries) versus mature vessels (defined as voluminous linear filamentous capillaries); (2) presence of an anastomotic arcade (peripheral connection between tiny branching vessels); (3) presence of loops (inner anastomoses between tiny vessels); and (4) presence of perilesional hypointense halo (the hypointense area is considered as a region of choriocapillaris alteration, corresponding to local flow impairment). Criteria were graded in a binary method ( 1 for presence and 0 for absence).

All structural OCT and OCT-A images were separately evaluated 1 month apart from each other by two masked experienced ophthalmologists (FC and $\mathrm{CF}$ ). Their evaluation aimed to accurately determine the morphological features of a CNV on OCT angiograms and the presence of exudation on structural OCT (figures 1 and 2). This process was useful in evaluating the intergrader reproducibility. All images were analysed independently

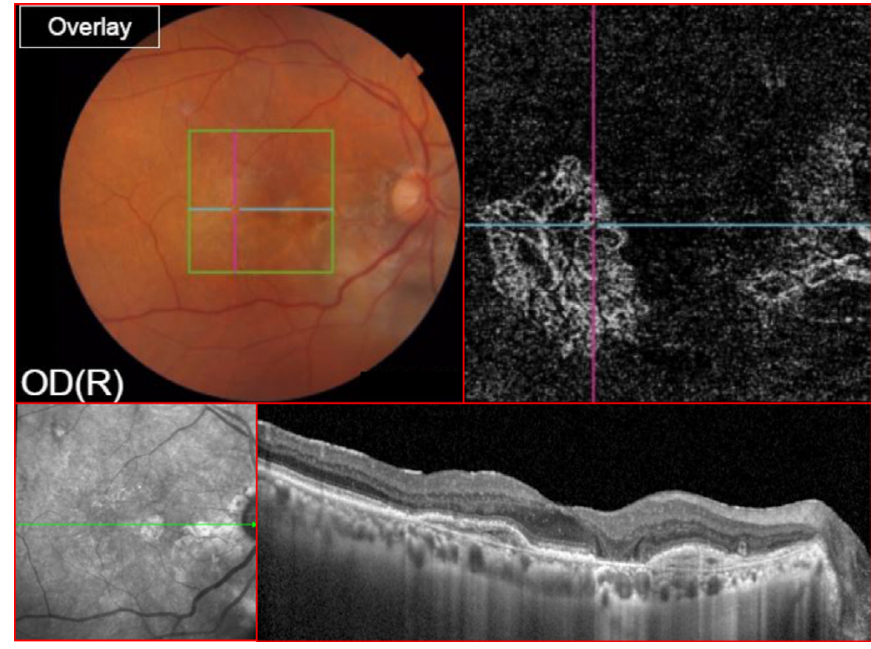

Figure 1 Multimodal imaging of a 75-year-old patient suffering from neovascular age-related macular degeneration and followed up for 15 months. The visual acuity after nine anti-vascular endothelial growth factor (VEGF) intravitreal injections was 32 ETDRS letters. Colour fundus photo (top left) shows the area examined on optical coherence tomography (OCT) angiography (green square). The 'en-face' OCT angiogram (top right), segmented at the level of the pigmented epithelium detachment, shows type 1 choroidal neovascularisation (CNV) with a well-visible peripheral anastomotic arcade. There is no space between all inner branching capillaries. This CNV shows some features that are generally associated with an active (exudative) lesion such as tiny branching vessels and peripheral arcade. The cross-sectional OCT of the same eye does not show any sign of fluid accumulation (bottom).

in a separate sitting to evaluate intragrader reproducibility. The same evaluation was then carried out by a third observer (RS), from a different ophthalmological centre, on a validation sample from a different group of patients but with the same inclusion and exclusion criteria.

\section{Statistical analysis}

Descriptive statistics (mean \pm SD or frequencies and percentages) were used to analyse all variables. For qualitative variables, intragrader and intergrader kappa coefficients were accessed by using the $\chi^{2}$ test on a contingency table. Agreement between masked observers was assessed using the kappa statistic. A learning sample was used to develop a model that describes the relation between the qualitative variables and the variable answer and a validation sample to test the developed statistical model with another observer and different patients. The outcome assessment consisted of the decision to retreat with IVI after structural OCT evaluation.

The statistical analysis included an investigation of association between the variable of interest and the potential predictors (univariate analysis). Principal component analysis with logistic regression analysis was performed to predict the effect of the four variables on a binary response variable. A set of $p$ uncorrelated factors and normalised coefficients was built with values of correlation between the explanatory variable (OCT-A criteria) and the variable answer (retreatment decision), to minimise the bias related to their multicollinearity. The relative risk for each OCT-A criterion was calculated. Receiver operating characteristic (ROC) analysis of the predictive model was issued. A p value $<0.05$ was considered statistically significant. All demographic, clinical and structural OCT/OCT-A measurements were 


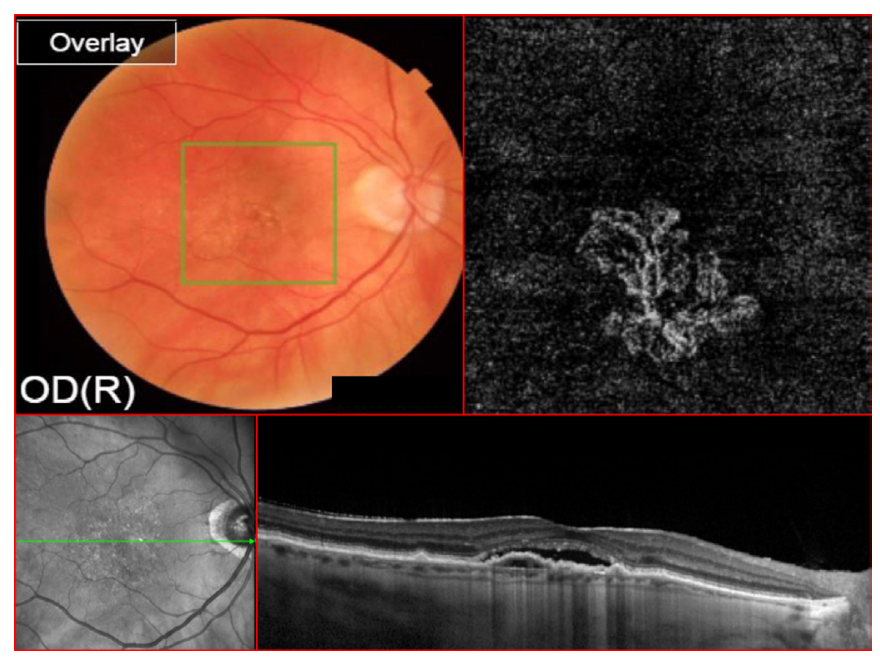

Figure 2 Multimodal imaging of an 80-year-old patient suffering from neovascular age-related macular degeneration and followed up for 18 months. The visual acuity after 12 anti-vascular endothelial growth factor (VEGF) intravitreal injections was 27 ETDRS letters. Colour fundus photo (top left) shows the area examined on optical coherence tomography (OCT) angiography (green square). The 'en-face' OCT angiogram (top right), segmented at the level of the pigmented epithelium detachment, shows type 1 choroidal neovascularisation (CNV) with a well-visible network of tiny branching vessels and loops. There is no space between all inner branching capillaries. This CNV shows some features that are generally associated with an active (exudative) lesion such as tiny branching vessels. The cross-sectional OCT of the same eye shows subretinal fluid accumulation (bottom).

tabulated in Microsoft Excel 2013 software from Microsoft. All statistics were performed through XLSAT 2017 (Data Analysis and Statistical Solution for Microsoft Excel, V.2017.3).

\section{RESULTS}

One-hundred and twenty-six eyes of 126 consecutive patients with exudative eAMD were enrolled in the current study. All subjects were Caucasian (97 females, 77\%) with a mean age of $79.5 \pm 8.9$ years. Baseline demographical and clinical characteristics are summarised in table 1.

Fluid was identified in 101 eyes (80\%). Mean CMT was $319.6 \pm 112.5 \mu \mathrm{m}$.

Table 1 Demographic and clinical characteristics of patients during anti-VEGF intravitreal injection treatment

\begin{tabular}{|ll|}
\hline Total eyes & 126 \\
\hline Patients, $n(\%)$ & 126 \\
\hline \multicolumn{1}{|c|}{ Female } & $97(77)$ \\
\hline Male & $29(33)$ \\
\hline Age, mean \pm SD (years) & $79.5(8.9)$ \\
\hline Mean BCVA, mean \pm SD (ETDRS letters) & $63.7(14.8)$ \\
\hline Follow-up time, mean \pm SD (months) & $22.9(13.5)$ \\
\hline Previous anti-VEGF treatment, mean \pm SD, n & $10.4(6.5)$ \\
\hline IVI interval, mean $\pm S D$ (months) & $2.35(1.42)$ \\
\hline CNV classification, $n$ (\%) & $107(85)$ \\
\hline Type 1 & $21(15)$ \\
\hline Type 2
\end{tabular}

BCVA, Best Corrected Visual Acuity; CNV, choroidal neovascularisation; IVI, intravitreal injection; VEGF, vascular endothelial growth factor.
Table 2 Sensitivity and specificity of each optical coherence tomography angiography feature of choroidal neovascularisation

\begin{tabular}{lllll}
\hline & Tiny branching & Arcade & Loops & Halo \\
\hline Sensitivity (\%) & 94.4 & 78.9 & 79.6 & 62.8 \\
Specificity (\%) & 47.2 & 63.9 & 91.3 & 64.9 \\
PPV (\%) & 81.7 & 84.5 & 97.6 & 76.2 \\
NPV (\%) & 77.3 & 54.8 & 50 & 88.1 \\
FP rate & 0.53 & 0.36 & 0.09 & 0.07 \\
FN rate & 0.06 & 0.21 & 0.20 & 0.35 \\
\hline
\end{tabular}

FN, false negative; FP, false positive; NPV, Negative Predictive Value; PPV, Positive Predictive Value.

The decision to treat and mean CMT were positively correlated in a statistically significant manner $(\mathrm{p}<0.002)$.

In OCT-A image analysis, the occurrence of the four different parameters was distributed as follows: tiny branching vessels in $82.5 \%$ (104 eyes), vascular loops in $81.7 \%$ (103 eyes), a peripheral anastomotic arcade in $66.7 \%$ (84 eyes) and a hypointense choriocapillaris halo in 54.8\% (69 eyes). The intragrader kappa coefficient was $88.5 \%$ and the intergrader kappa coefficient was $78 \%$. The concordance rate on the validation test from the third grader (RS) on 104 similar records of retreated CNV was $90.9 \%$.

In the univariate analysis, there was a weak association between IVI number and the presence of a peripheral arcade $(r=0.171, p=0.04)$ and an absence of an association between IVI number and tiny branching vessels $(p=0.071)$, vascular loops $(p=0.22)$ or a hypointense choriocapillaris halo $(p=0.064)$. All the four different OCT-A criteria showed a statistically significant $(\mathrm{p}<0.0001)$ correlation with an active lesion on structural OCT. Sensitivity, specificity and positive and negative predictive values were independently assessed for each qualitative parameter. False positive and false negative rates were also independently assessed for all qualitative parameters (table 2).

In the overall analysis, considering all four OCT-A criteria, sensitivity was $96.7 \%$ and specificity was $66.7 \%$. The false positive and negative rates were $33.3 \%$ and $3.3 \%$, respectively. Considering the $71.4 \%$ prevalence of eAMD in our study, the positive predictive value was $87.9 \%$, and the negative predictive value was $88.9 \%$.

On multivariate analysis, the combination of all the four OCT-A criteria predicted the exudative status of the CNV lesion in $97.6 \%$ of cases. The ROC curve of a model including the four initial variables (OCT-A criteria) predicts the status of the lesion in $94.4 \%$ (figure 3 ). The cluster of tiny branching network and the peripheral arcade on OCT-A predicted a positive outcome (exudative status of the CNV) for two OCT-A criteria (tiny branching and peripheral arcade) in $71.23 \%$ of cases.

The principal component analysis demonstrated the strength of the link between the explanatory variable (OCT-A criteria) and the variable answer (exudative status of the CNV and the consequential decision of treatment) for peripheral arcade, loops, tiny branching vessels and hypointense halo with a relative risk of $2.41,2.96,6.76$ and 1.73 , respectively (table 3 ).

\section{DISCUSSION}

In the current study, we tried to validate new criteria for treating patients with eAMD based on OCT-A-based CNV qualitative findings and compared these with the well-established structural OCT outcomes. This morphological CNV assessment was previously reported by Coscas et al in a pilot study of 73 consecutive patients with AMD. ${ }^{11}$ 


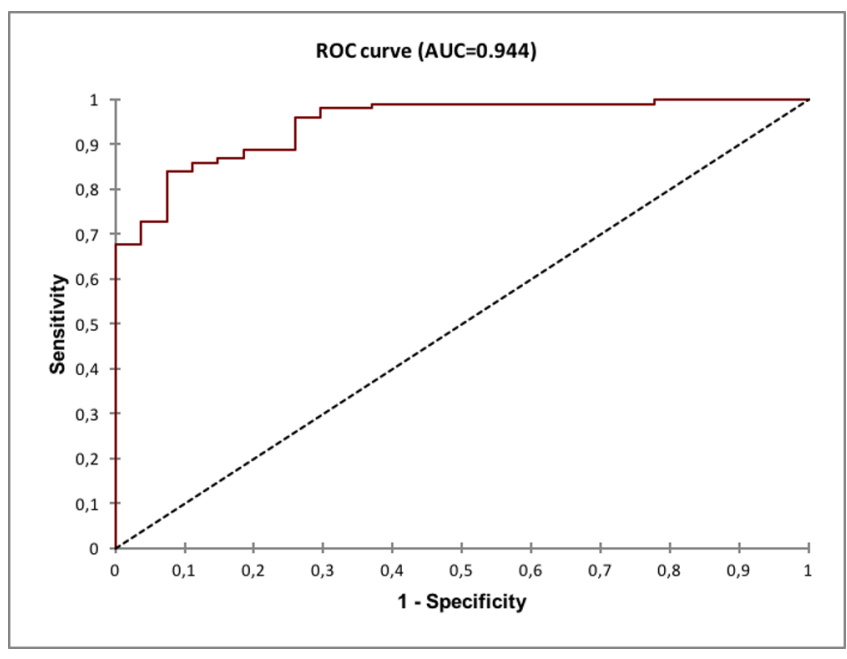

Figure 3 The receiver operating characteristic (ROC) curve between sensitivity and specificity showed that the current model, based on optical coherence tomography angiography findings, correctly predicts the active status of a choroidal neovascularisation (AUC, area under the curve) with a high probability for a therapeutic decision in each patient.

The probability of IVI treatment based on the presence of all four OCT-A criteria on OCT-A was $97.6 \%$. The criterion 'tiny branching vessels' had the best correlation value with treatment decision and the highest relative risk. The criterion 'halo' had a non-statistically significant link with the decision of treatment and a low relative risk. In clinical activity, it is common practice to cluster criteria in pairs to easily summarise morphological changes. According to our analysis, tiny branching vessels and the peripheral arcade are the OCT-A criteria with the highest relative risk of exudative signs on structural OCT. Using a model with normalised coefficients, we demonstrated that the probability of IVI treatment considering tiny branching vessels and the peripheral arcade on OCT-A is $71.23 \%$, which is inferior to a model containing the four criteria.

These two criteria were also suggested as important biomarkers by Al-Sheikh et $a^{16}$ in a study of 31 eyes including 11 eyes with active lesions and 20 eyes with quiescent lesions. Tiny branching vessels were present in $82 \%$ of active lesions but in only $30 \%$ of quiescent ones $(\mathrm{p}<0.05)$. The peripheral arcade was also present in $82 \%$ of the active lesions compared with only $40 \%$ of the quiescent ones. This huge difference was not statistically

\begin{tabular}{|c|c|c|c|c|c|}
\hline CNV features & Values & Lower bound (95\%) & Upper & bound $(95 \%)$ & $P$ values \\
\hline Arcade & 0.575 & 0.254 & 0.896 & & 0.000 \\
\hline Loops & 1.322 & 0.517 & 2.127 & & 0.001 \\
\hline Tiny branching & 1.207 & 0.627 & 1.788 & & $<0.0001$ \\
\hline Halo & 0.078 & -0.717 & 0.873 & & 0.848 \\
\hline CNV features & $\mathbf{R R}$ & \multicolumn{2}{|c|}{ Lower bound (95\%) } & \multicolumn{2}{|c|}{ Upper bound (95\%) } \\
\hline Arcade & 2.41 & 1.68 & & \multicolumn{2}{|l|}{3.46} \\
\hline Loops & 2.93 & 1.62 & & \multicolumn{2}{|l|}{5.31} \\
\hline Tiny branching & 6.76 & 2.58 & & \multicolumn{2}{|l|}{17.75} \\
\hline Halo & 1.73 & 1.37 & & \multicolumn{2}{|l|}{2.17} \\
\hline
\end{tabular}

Values of the explanatory variables of the statistical mode.

Value: strength of the link between the explanatory variable (criteria) and the variable answer (decision of retreatment).

CNV, choroidal neovascularisation; $\mathrm{RR}$, relative risk significant probably due to the small sample size and consequent lack of adequate power. Nevertheless, these criteria may be considered as potential biomarkers of CNV activity.

The biological basis of these criteria could be related to the angiogenetic process. The prominent vessel is covered by pericytes and appears to be resistant to anti-VEGF therapy whereas the tiny branching capillaries, sprouting from the periphery of the CNV, are more vulnerable due to the lack of pericytes. Moreover, since the appearance of pericytes on vessel walls is a later process than the angiogenetic one, it can be associated with new-onset, immature capillaries, therefore potentially linked with the exudative status of a neovascular lesion. ${ }^{1718}$

We showed a high rate $(12 / 36 ; 33.3 \%)$ of false positive qualitative evaluations on OCT-A (ie, potentially active CNV according to the presence of OCT-A criteria but inactive according to the OCT B-scan). When assessing the entire patient sample (false positive + false negative ones) this rate dropped to $9.52 \%$. This ratio could be explained with a potential superiority of OCT-A criteria, compared with structural OCT, in enabling the earlier detection of CNV activity, before the exudation occurs. The aim of early detection is to enable a prompt treatment in order to preserve the macular architecture and, consequently, the visual acuity. Several studies on eAMD showed that a decreased visual acuity is a late sign of activity and, currently, we mostly treat patients according to structural OCT signs, rather than BCVA, according to the principle: the sooner is the fluid detection, the better is the final visual acuity. ${ }^{19}$

Every imaging modality suffers from some limitations. We observed some false negative patients on OCT-A. These cases corresponded mainly to patients with pigment epithelium detachment (PED) or haemorrhage with potential difficulties in imaging the CNV due to attenuation or blockage of the signal.

Several previous studies, using different devices and different decorrelation algorithms (spectral domain or swept-source OCT), reported that the sensitivity of OCT-A in detecting choroidal neovascular lesions ranged from 50\% to $87 \% .^{79}{ }^{20}$ In a recent prospective study of 182 eyes, Eandi et al demonstrated a good agreement $(\mathrm{r}=0.84)$ between ICGA and OCT-A for CNV size, with a higher percentage of well-defined lesion borders detected by OCT-A. ${ }^{21}$ However, the agreement between these techniques was only moderate for the definition of CNV borders (kappa $=0.4) .{ }^{21}$ We used two masked experienced readers and a validation test to minimise any resulting bias: as a result the intergrader correlation coefficient was very good.

The study patient population includes only patients who were previously treated with anti-VEGF agents. However, according to previous studies, there is only subtle change after anti-VEGF treatment and, in case of recurrence, the CNV lesion regains its original morphology. ${ }^{17} 202223$ Large prominent vessels in fact are not affected by anti-VEGF treatment whereas the tiny vessels are attenuated and pruned.

Potential limitations of the current study are its retrospective nature and the relatively high prevalence of patients with an active lesion in which OCT-A criteria were tested. We acknowledge that treatment-naïve eyes or quiescent lesions were not enrolled. Further studies including a larger proportion of treatment-naïve and quiescent CNVs might be performed in order to validate the negative predictive value of our study criteria. Moreover, current results are based on qualitative criteria assessed by OCT-A while quantitative methods could be more reliable. The qualitative OCT-A analysis has its own limitations characteristic of all binary qualitative systems; for example, even if two networks have the same qualitative evaluation, they may have different degrees of activity that might 
impact the treatment decision and follow-up. Therefore, quantification of the extent of neovascular networks is a task of major importance, ${ }^{15}$ although quantitative representations of neovascular networks are often subjective and rely on manual delineation. ${ }^{2024}$ Proven methods for automated detection and quantification of CNV are lacking, mostly due to projection and foreground imaging artefacts. ${ }^{25}$ Future research may shed some light on the quantification of the size, density and complexity of neovascular networks and their correlation with the decision to treat and response to treatment.

In conclusion, OCT-A appears to be a useful technique in guiding the decision to treat and following up patients with eAMD, and that could be routinely used in clinical practice. A qualitative assessment of the CNV, based on OCT-A criteria, may substantially increase the capability to detect early recurrences, leading to a proactive therapeutic strategy, and providing the clinician additional parameters to establish treatment decisions and monitoring in eAMD.

Contributors $\mathrm{FC}$ and $\mathrm{ML}$ provided a substantial contribution to the conception of the work, to the acquisition, analysis and interpretation of data, in drafting the manuscript and in giving the final approval of the submitted study. FC and ML equally contributed to the manuscript and may be both considered as firs author. JFB, AS, DC, RS and CF contributed to the acquisition, analysis and interpretation of data and gave the final approval of the submitted study. EHS and GC provided a substantial contribution in the conception of the work, in drafting the manuscript and gave the final approval of the submitted study.

Funding The authors have not declared a specific grant for this research from any funding agency in the public, commercial or not-for-profit sectors.

Competing interests None declared.

Patient consent Obtained.

Ethics approval Paris Institutional Ethics Committees (IEC).

Provenance and peer review Not commissioned; externally peer reviewed.

Open access This is an open access article distributed in accordance with the Creative Commons Attribution Non Commercial (CC BY-NC 4.0) license, which permits others to distribute, remix, adapt, build upon this work non-commercially, and license their derivative works on different terms, provided the original work is properly cited, appropriate credit is given, any changes made indicated, and the use is non-commercial. See: http://creativecommons.org/licenses/by-nc/4.0

\section{REFERENCES}

1 Bressler NM. Age-related macular degeneration is the leading cause of blindness.... JAMA 2004;291:1900-1.

2 Gess AJ, Fung AE, Rodriguez JG. Imaging in neovascular age-related macular degeneration. Semin Ophthalmol 2011;26:225-33.

3 Sulzbacher F, Kiss C, Munk M, et al. Diagnostic evaluation of type 2 (classic) choroidal neovascularization: optical coherence tomography, indocyanine green angiography, and fluorescein angiography. Am J Ophthalmol 2011;152:799-806.

4 Musa F, Muen WJ, Hancock R, et al. Adverse effects of fluorescein angiography in hypertensive and elderly patients. Acta Ophthalmol Scand 2006;84:740-2.
5 Lalwani GA, Rosenfeld PJ, Fung AE, et al. A variable-dosing regimen with intravitreal ranibizumab for neovascular age-related macular degeneration: year 2 of the PrONTO Study. Am J Ophthalmol 2009;148:43-58

6 Spaide RF, Klancnik JM, Cooney MJ. Retinal vascular layers imaged by fluorescein angiography and optical coherence tomography angiography. JAMA Ophthalmol 2015;133:45-50

7 Jia Y, Bailey ST, Wilson DJ, et al. Quantitative optical coherence tomography angiography of choroidal neovascularization in age-related macular degeneration. Ophthalmology 2014;121:1435-44.

8 Adhi M, Liu JJ, Qavi AH, et al. Choroidal analysis in healthy eyes using swept-source optical coherence tomography compared to spectral domain optical coherence tomography. Am J Ophthalmol 2014;157:1272-81.

9 Moult E, Choi W, Waheed NK, et al. Ultrahigh-speed swept-source OCT angiography in exudative AMD. Ophthalmic Surg Lasers Imaging Retina 2014;45:496-505.

10 Inoue $\mathrm{M}$, Jung JJ, Balaratnasingam C, et al. A comparison between optical coherence tomography angiography and fluorescein angiography for the imaging of type 1 neovascularization. Invest Ophthalmol Vis Sci 2016;57:0CT314-0CT323.

11 Coscas GJ, Lupidi M, Coscas F, et al. Optical coherence tomography angiography versus traditional multimodal imaging in assessing the activity of exudative agerelated macular degeneration: a new diagnostic challenge. Retina 2015;35:2219-28.

12 Freund KB, Zweifel SA, Engelbert M. Do we need a new classification for choroidal neovascularization in age-related macular degeneration? Retina 2010;30:1333-49.

13 Holz FG, Amoaku W, Donate J, et al. Safety and efficacy of a flexible dosing regimen of ranibizumab in neovascular age-related macular degeneration: the SUSTAIN study. Ophthalmology 2011;118:663-71.

14 Liakopoulos S, Ongchin S, Bansal A, et al. Quantitative optical coherence tomography findings in various subtypes of neovascular age-related macular degeneration. Invest Ophthalmol Vis Sci 2008;49:5048-54.

15 Schmidt-Erfurth U, Chong V, Loewenstein A, et al. Guidelines for the management of neovascular age-related macular degeneration by the European Society of Retina Specialists (EURETINA). Br J Ophthalmol 2014;98:1144-67.

16 Al-Sheikh M, lafe NA, Phasukkijwatana N, et al. Biomarkers of neovascular activity in age-related macular degeneration using optical coherence tomography angiography. Retina 2018;38:220-30.

17 Spaide RF. Optical coherence tomography angiography signs of vascular abnormalization with antiangiogenic therapy for choroidal neovascularization. Am J Ophthalmol 2015;160:6-16.

18 Jain RK. Normalizing tumor vasculature with anti-angiogenic therapy: a new paradigm for combination therapy. Nat Med 2001;7:987-9.

19 Hoerster R, Muether PS, Hermann MM, et al. Subjective and functional deterioration in recurrences of neovascular AMD are often preceded by morphologic changes in optic coherence tomography. Br J Ophthalmol 2011;95:1424-6.

20 de Carlo TE, Bonini Filho MA, Chin AT, et al. Spectral-domain optical coherence tomography angiography of choroidal neovascularization. Ophthalmology 2015; 122:1228-38.

21 Eandi CM, Ciardella A, Parravano M, et al. Indocyanine green angiography and optica coherence tomography angiography of choroidal neovascularization in age-related macular degeneration. Invest Opthalmol Vis Sci 2017:58:3690.

22 Huang D, Jia Y, Rispoli M, et al. Optical coherence tomography angiography of time course of choroidal neovascularization in response to anti-angiogenic treatment. Retina 2015;35:2260-4

23 Lumbroso B, Rispoli M, Savastano MC. longitudinal optical coherence tomographyangiography study of type 2 naive choroidal neovascularization early response after treatment. Retina 2015;35:2242-51.

24 Kuehlewein L, Bansal M, Lenis TL, et al. Optical coherence tomography angiography of type 1 neovascularization in age-related macular degeneration. Am J Ophthalmol 2015; 160:739-48.

25 Zhang M, Hwang TS, Campbell JP, et al. Projection-resolved optical coherence tomographic angiography. Biomed Opt Express 2016;7:816-28. 